\title{
Resources Management using Adaptive Fade Mitigation Techniques in DVB-S2/RCS Multi-Beam Systems
}

\author{
Hartmut Brandt, Vincent Boussemart, Christian Kissling, Cristina Parraga Niebla \\ German Aerospace Center (DLR e.V.), Institute for Communications and Navigation, Oberpfaffenhofen, 82234 \\ Wessling, Germany \\ Oliver Lücke, Ricard Vilalta, Émeric Dupont \\ TriaGnoSys GmbH, Argelsrieder Feld 22, 82234 Wessling, Germany \\ and \\ Robert Schweikert, Thomas Wörz \\ Audens ACT, Argelsrieder Feld 22, 82234 Wessling, Germany
}

\begin{abstract}
[Abstract] A powerful and flexible software radio resource management (RRM) simulator suited for modelling a realistic Ka-band multi-beam DVB-S2/RCS system with European coverage is described. The simulator enables design, optimisation and trade-off of RRM techniques that are suitable for the DVB-S2 and an enhanced adaptive DVB-RCS standards which allow efficiently counteracting the link quality variations and thus fully exploiting the adaptive physical layer resulting from the use of adaptive coding and modulation (ACM) while providing Quality of Service (QoS) support.
\end{abstract}

\begin{tabular}{|c|c|c|c|c|c|}
\hline \multirow[b]{2}{*}{$A C M$} & \multicolumn{4}{|c|}{ Nomenclature } & \multirow[b]{2}{*}{ Mega symbols per second } \\
\hline & $=$ & Adaptive Coding and Modulation & Msps & $=$ & \\
\hline BBFrame & $=$ & Baseband Frame & $O B P$ & $=$ & On-Board Processor or Processing \\
\hline CAC & $=$ & Call Admission Control & $P D F$ & $=$ & Probability Density Function \\
\hline$C D F$ & $=$ & Cumulative Distribution Function & $P E P$ & $=$ & Policy Enforcement Point \\
\hline \multirow[t]{2}{*}{ CEMS } & $=$ & Channel Estimator and Modcod & $P L$ & $=$ & Physical Layer \\
\hline & & Selector & POP3 & $=$ & Post Office Protocol version 3 \\
\hline$C / I$ & $=$ & Carrier-to-Interference ratio & QoS & $=$ & Quality of Service \\
\hline$D V B-S$ & $=$ & Digital Video Broadcast over Satellite & NCC & $=$ & Network Control Centre \\
\hline FCT & $=$ & Frame Composition Table & $N P R$ & $=$ & Noise Power Ratio \\
\hline FDM & $=$ & Frequency Division Multiplex & $R C S$ & $=$ & Return Channel over Satellite \\
\hline FEC & $=$ & Forward Error Correction & $R L C C$ & $=$ & Return-Link Congestion Control \\
\hline FLCC & $=$ & Forward-Link Congestion Control & $R M$ & $=$ & Resource Manager or Management \\
\hline$F M T$ & $=$ & Fade Mitigation Techniques & $R R M$ & $=$ & Radio Resource Management \\
\hline FSS & $=$ & Fixed Satellite Service & SCT & $=$ & Superframe Composition Table \\
\hline$G S$ & $=$ & Generic Stream & $S I$ & $=$ & Service Information \\
\hline GSE & $=$ & Generic Stream Encapsulation & SLA & $=$ & Service Level Agreement \\
\hline$G W$ & $=$ & Gateway & SNIR & $=$ & Signal-to-Noise-and-Interference- \\
\hline$H P A$ & $=$ & High-Power Amplifier & & & Ratio \\
\hline IP & $=$ & Internet Protocol & TBTP & $=$ & Terminal Burst Time Plan \\
\hline IWF & $=$ & Inter-Working Function & TCT & $=$ & Time-slot Composition Table \\
\hline$M A C$ & $=$ & Medium Access Control & $T D M$ & $=$ & Time Division Multiplex \\
\hline$M F-T D M A$ & $=$ & Multi-Frequency TDMA & TDMA & $=$ & Time Division Multiple Access \\
\hline \multirow[t]{2}{*}{$M P E$} & & Multi-Protocol Encapsulation & VoIP & $=$ & Voice over IP \\
\hline & & & $X M L$ & $=$ & Extensible Markup Language \\
\hline
\end{tabular}




\section{Introduction}

$\mathrm{T}$ HE second generation Digital Video Broadcasting for Satellite standard ETSI EN 302307 (DVB-S2) ${ }^{1}$ incorporates the application of Fade Mitigation Techniques (FMT) for the forward link by use of Adaptive Coding and Modulation (ACM). Research on adaptive techniques to optimise the Return Channel over Satellite (RCS) link capacity is not yet standardised and on-going. ${ }^{2}$

Besides other key technologies such as use of higher frequency bands at K/Ka-band and multi-beam antenna patterns, in particular ACM has been considered as a powerful tool to increase further system capacity by better modulation and coding efficiency which is dynamically adjusted to the individual terminal requirement. Thus, ACM is included in the DVB-S2 standard as normative for the interactive application area.

A capacity gain by a factor of about 2.5 is expected to be achieved with DVB-S2 compared to a conventional, non-adaptive Time Division Multiplex (TDM) system, considering a realistic Ka-band multi-beam system with European coverage. ${ }^{3,4}$

This paper presents work performed within the ESA funded project on "Resources Management using Adaptive Fade Mitigation Techniques (FMT) in DVB-RCS Multi-Beam Systems”." A powerful and flexible software radio resource management (RRM) simulator is currently being developed in the frame of this project. It will allow to design, optimise and trade-off RRM techniques that are suitable for the DVB-S2 and DVB-RCS standards, efficiently counteracting the link quality variations and thus fully exploiting the adaptive physical layer resulting from the use of ACM while providing Quality of Service (QoS) support. The IP and RRM relevant signalling traffic (e.g. Terminal Burst Time Plan (TBTP), channel estimation messages) of one or more spot beams containing hundreds to thousands of user terminals will be simulated using the software tool. Simulation of both a transparent as well as a regenerative satellite scenario is possible.

The organisation of the paper is as follows.

Section II presents the DVB-S2/RCS multi-beam system modelled in the simulator.

Section III focuses on the RRM techniques that are investigated using the simulator. Novel RRM algorithms are developed for optimum use of the capabilities of ACM and will be verified with the simulator to efficiently provide QoS to delay or loss sensitive applications (e.g. for Voice-over-IP) despite the capacity variations caused by the changing link quality due to possibly extensive rain fading events. Of central interest is the development of scheduling algorithms providing an optimum balance between QoS and efficient use of capacity and, further, the development of efficient protocols for transmission of IP data over S2 and adaptive RCS.

Section IV presents a detailed description of the simulator architecture and the implemented DVB-S2/RCS system model, where an enhanced adaptive RCS scheme is also shortly described.

Finally, simulation results are presented in section V, showing the performance of the novel RRM techniques and protocols.

\section{System Outline}

In order to assess the performance of various resource management schemes that can exploit fade mitigation techniques, the two system scenarios (cf. Figure 1), described in the following, are specified:

- A system with a transparent/bent-pipe satellite, multi-beam coverage and a high speed forward and a low to medium data rate return link; a multi-star network configuration is assumed, i.e. each gateway supports the users in a certain number of beams, any link between users needs a double hop through the satellite (no inter-beam connectivity). Consumer and corporate users requesting multimedia content are assumed. Hence, bursty internet-like and, to a certain extent, real-time multimedia traffic can be expected.

- A satellite system with a small regenerative piggyback payload; the regenerative processor shall constitute a small on-board processing (OBP) piggyback to a transparent access payload (forward plus return-link processors). The OBP shall interface the transparent payload at Ka-band and shall offer mesh connectivity to a subset of the user beams (approximately 10 beams). The OBP only interfaces to user uplink and downlinks bandwidths and not to the gateway (GW) uplink and downlink bandwidths; also the network control centre (NCC) is located on ground. It is assumed that the system is used by professional users, who mainly transmit bulky real-time multimedia traffic and, additionally, bursty internet-like traffic

\footnotetext{
${ }^{*}$ ESA Study 18826/05/NL/US, 2005. The consortium consists of TriaGnoSys GmbH (project leader), Audens ACT, German Aerospace Center (DLR), and Nera.
} 

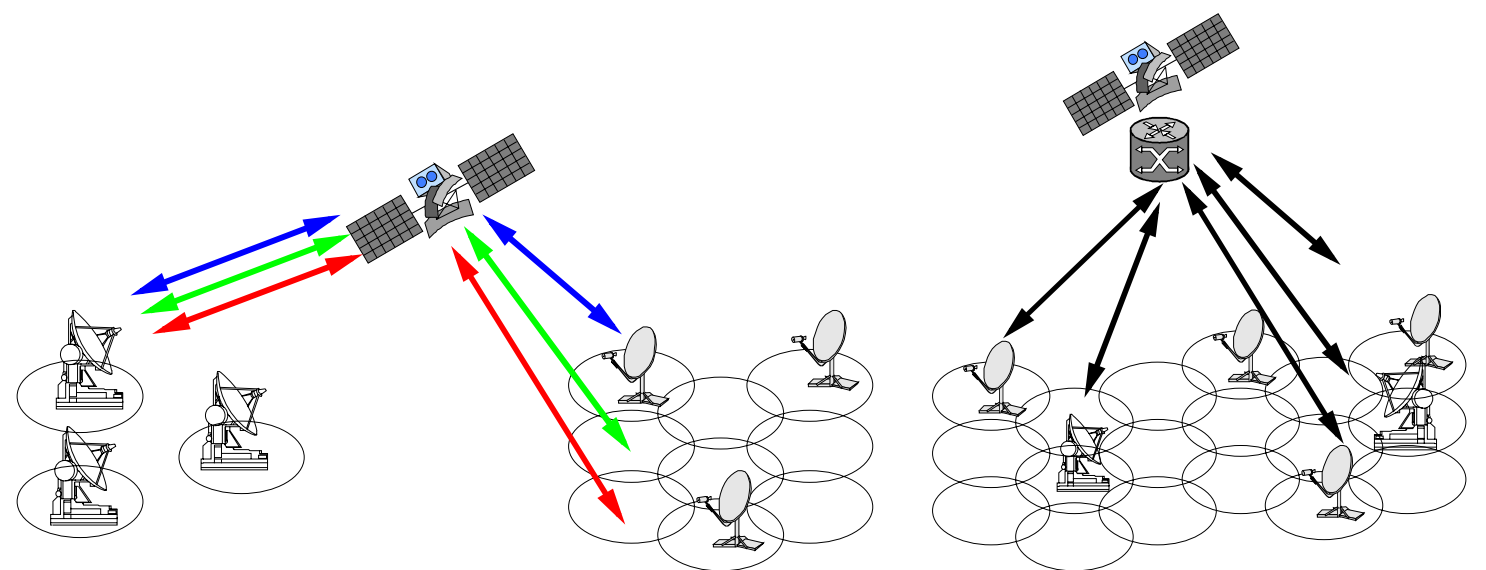

Figure 1: Transparent (left) and regenerative (right) satellite scenario

In both scenarios it is foreseen to utilise Ka-band although it is still considered a difficult business case but promising on the medium term due to the wider available spectrum, with fixed satellite services (FSS) allocation from $27.5-30.0 \mathrm{GHz}$ for uplink and $17.7-21.2 \mathrm{GHz}$ for the downlink, respectively.

In this paper the focus is on the transparent satellite scenario which is described next in detail.

The simulator is capable of simulating other user-defined scenarios than that being described in this subsection. However, the description of the particular scenario aims at providing insight into the system aspects that have to be taken into account in the simulator satellite system model.

A geostationary orbit at $33^{\circ}$ east is assumed for the satellite. The foreseen frequency bands are in agreement with the fixed satellite services (FSS) spectrum allocation:

- $27.5-29.5 \mathrm{GHz}$ and 19.7-20.2 GHz for the forward up-link (gateway to satellite), and down-link (satellite to user), respectively.

- $\quad$ 29.5-30.0 GHz and 17.7-19.7 GHz for the return up-link (user to satellite) and down-link (satellite to gateway), respectively.

The antenna has 72 spot beams and covers Europe including parts of the Middle East (cf. Figure 2). The antenna gain varies between $51-55 \mathrm{dBi}$ within a spot beam.

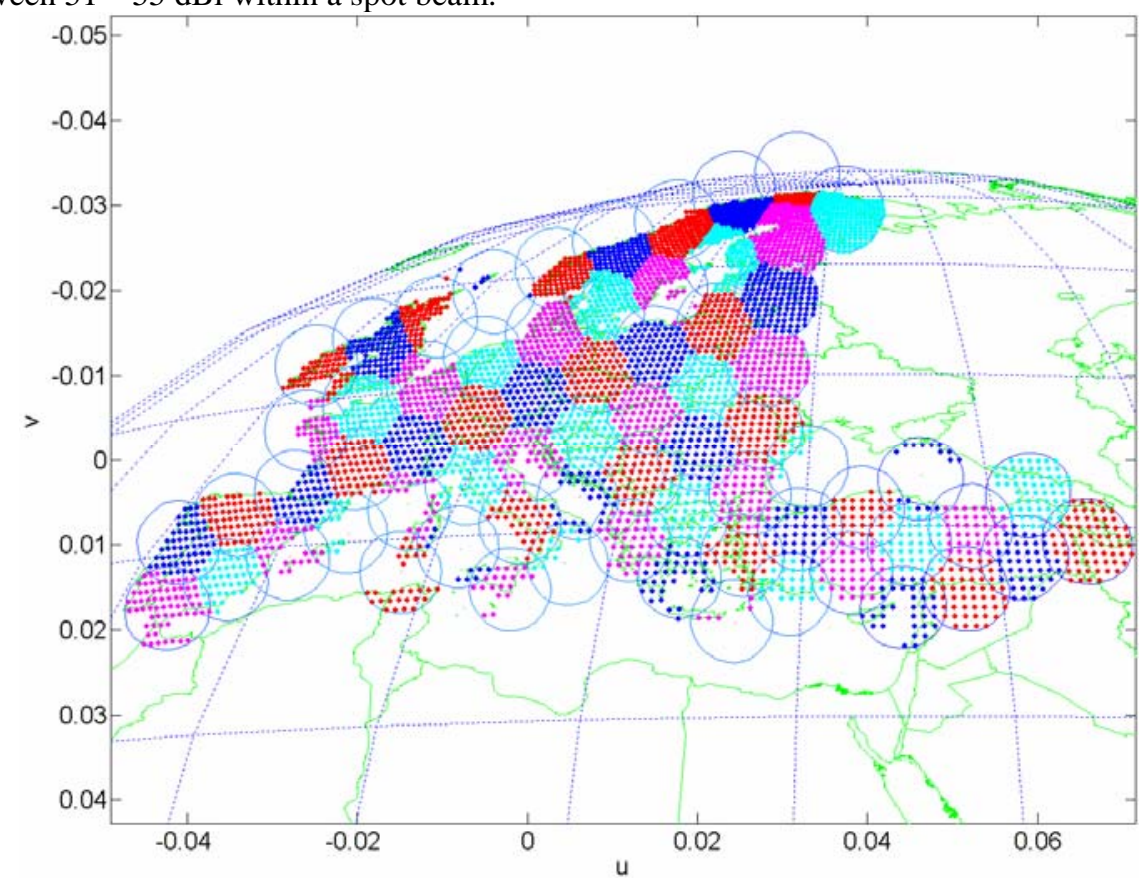

Figure 2: 72-spot beam pattern for European coverage. 
The systems employs a 4 colour frequency re-use scheme with polarisation re-use not being foreseen. A bandwidth of $125 \mathrm{MHz}$ is allocated for each user beam assuming a homogeneous resource distribution (i.e. each beam has the same bandwidth and satellite power allocation).

In the forward-link, using a 25\% roll-off and additional 11\% channel spacing, two carriers each with 45 Msps occupy the $125 \mathrm{MHz}$ bandwidth.

Three terminal classes with 1, 2, or 4 Msps are considered for the RCS return-link. The roll-off is increased to $35 \%$ and the channel spacing is kept at $11 \%$ with respect to the forward-link, leading to a bandwidth of 1.5 , 3.0, or 6.0 MHz per carrier. The actual number of carriers varies according to the terminal type distribution. In total, one approximately achieves $83.5 \mathrm{Msps}$ in a bandwidth of $125 \mathrm{MHz}$.

Five gateways serve the 72 beams, each being responsible for 14 or 15 beams (again, cf. Figure 2).

\section{RRM Techniques}

Here we describe what are the techniques implemented in the simulator, how it works. Three sub-sections will address resource management of the return-link, congestion control and call admission control, and scheduling techniques for the forward-link.

\section{A. Resource Management of the Return-Link}

The return link in DVB-RCS is organized in superframes (SF). ${ }^{2}$ As can be seen in the example in Figure 3, the SFs are structured in a Multi Frequency Time Division Multiple Access (MF-TDMA) scheme.

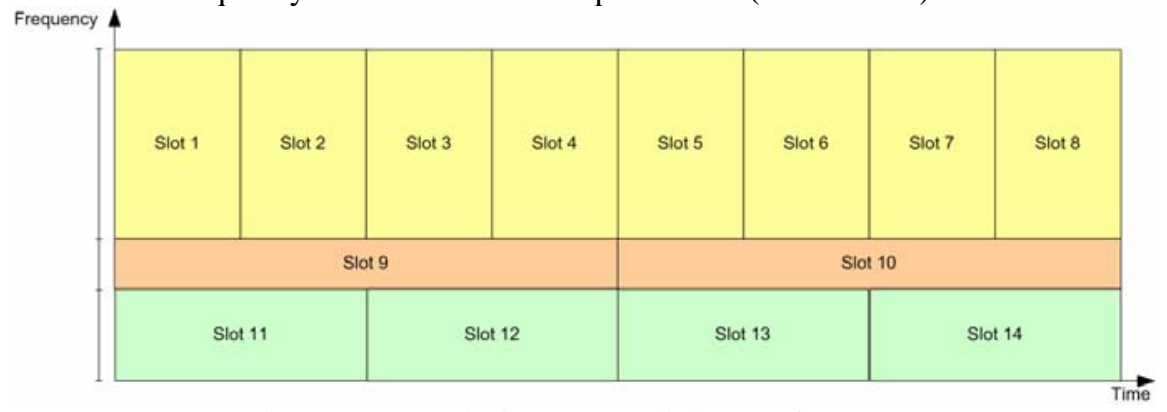

Figure 3 Example for a return link superframe.

Each terminal has to state a capacity request to the controlling gateway or NCC in order to receive capacity in one or several time slots. The return link Radio Resource Management module (RRM) processes all incoming requests and assigns time slots to the terminals. As mentioned above, an adaptive RCS is considered. ${ }^{5}$ Thus, for the use of adaptive coding and modulation as fade mitigation technique, it is additionally necessary to decide which combination of Modulation and Coding (ModCod) is used in each time slot. The decision which ModCod is the optimum for a terminal is taken based on the Channel Estimator and Modcod Selector (CEMS) methods (cf. Sec. IV-B). Within this work the following two different scenarios for the assignment of ModCods to time slots are considered. In the first scenario a ModCod can be dynamically assigned to each time slot. Here the binding of the used ModCod to a time slot can be different for every time slot and can also change for every SF. For this reason this scenario is referred to as dynamic scenario.

The second scenario assumes that for each time slot always a specific ModCod is used. In this case the binding of a ModCod to a time slot is static. Nevertheless it is possible here to provide a set of SFs whereas each SF can contain a different set of ModCods. The RRM module then has the possibility to select one SF from this set for the actual allocation round, which suits the actual channel states and capacity demands best. In the subsequent allocation rounds it is possible to also select a different SF from the set. In this case only the selection of a SF is dynamic but the binding of a ModCod to a time slot within each SF is static and cannot be changed on the fly. This scenario is denominated as semi-dynamic for this reason. The RRM algorithm here has to additionally consider, that a terminal must be assigned either the optimum ModCod or a more robust one, since the BER would increase drastically otherwise.

The algorithms implemented in the RRM module additionally consider fairness of the assignments, provision of a high degree of QoS (e.g. minimization of delay and delay jitter), conflict resolution (in case more capacity is demanded than available) and maximization of the spectral efficiency. ${ }^{7}$

\section{B. Congestion Control and Call Admission Control}


The task of the Congestion Control is preventing congestion and, if this is not possible, minimizing the impact of congestion situations when they occur and foster fast recovery. Mechanisms are utilized to first detect the presence of congestion and then to recover the stability of the system. The mechanisms used for the return- and for the forward-link are not identical since the traffic is asymmetric and thus the usage of the links is different.

On one hand, the presence of congestion in the forward-link can be detected by analysing the state of the packet pools in the scheduler. When the number of packets dropped because of a timeout or because of an excess of capacity reaches a given amount this is an indication of congestion. The thresholds used to set the congestion indication flag are directly related to the type of the pool, each pool being attributed to a particular class (gold or silver) and to a particular Quality of Service (QoS) - expedited forwarding, assured forwarding or best-effort . The congestion detection flag is set in correlation with the congestion indication when the arrival rate of a given pool is exceeding the serving rate. In that case mechanisms are started in order to resolve the congestion: the Call Admission Control (CAC) functionality is disabled meaning that all CAC Requests will automatically be rejected and dropping rates are set in the pools. The dropping can be either randomly distributed between the different pools or QoS-dependant. If the congestion is not solved after having applied these mechanisms, i.e. if the packets are still dropped in the packet pools above specific thresholds, then the last strategy consists in switching to the backup Service Level Agreement (SLA). This will set more restrictive parameters at the Policy Enforcement Point (PEP) in order to directly reduce the incoming traffic. Once there is no more congestion each mechanism is switched back to its default state.

On the other hand, the presence of congestion in the return-link is detected in the gateway and in the RCS terminals. In the gateway congestion is indicated by the resource manager itself when the resources requested cannot be all allocated. In the terminal congestion is detected by analysing the state of the queues present in the scheduler (dropping rates). When congestion is indicated by the gateway the Call Admission Control (CAC) functionality is disabled and the backup SLA is switched on in the corresponding terminal. When congestion is indicated by the terminal itself dropping rates are applied to the queues present in the scheduler. In the same way once the congestion ended all mechanisms are switched off.

The Call Admission Control technique is utilized together with particular types of applications (mainly real-time applications) to guaranty resources for the transmission. It consists in an exchange of messages between the entity requesting resources and the entity which is aware of them. First a request is transmitted and contains the amount of resources needed (in terms of bandwidth for instance). At the reception of this request algorithms are started in order to determine if the resources requested by the entity can be supported by the system or not. In the case of Voice over IP the traffic is bi-directional and symmetric meaning that resources have to be requested for both the return- and the forward-links. If both links can provide the support of the resources then the request is accepted, if not then it is rejected. The decision is taken by analysing separately the load of both forward- and return-links and by taking into account the ModCods which are currently assigned. The algorithms are inhibited when the CAC functionality is disabled, i.e. when congestion is detected in at least one of the two links. On the forward-link the load computations are based on the load of the pools in the scheduler and of the frame utilization in the modem. On the return-link the computations are mainly realized according to the number of free slots in the super frame.

\section{Scheduling techniques for the Forward-Link}

The packet scheduler in the forward link is responsible for sorting the packets coming from upper layers for transmission, obeying QoS criteria as well as transmission efficiency maximization for efficient capacity utilization. The scheduling technique implemented in the simulator is based on the so-called "group-efficient" scheduler proposed in Ref. 7, which guarantees that the ModCod information related to each packet is kept up to date. This is possible by keeping the actual ModCod information per terminal in an independent look-up table that is updated whenever a ModCod switch occurs and linking this information to the packets via pointers instead of classifying the packets according to their ModCod at the time they arrive at the system. The incoming packets are sorted according to their DiffServ QoS class and their SLA class in different packet pools, as can be observed in Figure 4; an additional pool with the highest priority holds MPEG packets which contain signalling information.

A number of packets from those backlogged in the pools are selected for transmission in each scheduling round, which is internally triggered by two trigger signals. One trigger is a timer that ensures that a new scheduling round is started not later than a fixed time after the last and the other trigger comes from the S2-Modem which tells the scheduler that it is going idle in the near future. The criteria to select packets in each scheduling round are different for each packet pool. ${ }^{7}$ In particular, the following criteria are applied in this implementation:

- EF pools: all backlogged packets at the beginning of the scheduling round are selected for transmission.

- AF pools: all backlogged packets exceeding an established queuing delay are selected for transmission. 
- $\quad$ BE pools: from the backlogged packets in the pool, those that fit the remaining resources after selecting $\mathrm{EF}$ and AF packets are selected for transmission.

At the beginning of a scheduling round, all selected packets are forwarded to the Layer-2 encapsulation and fragmentation module that corresponds to the actual ModCod of each packet, producing the data fields of BBFrames, the length of which depends on the ModCod. It should be noted that the ModCod of each packet is fetched from the terminal table when the packet is selected. After this a second round is run in which the pools are allowed to fill the scheduling interval with new frames according to pool priorities.

After the second scheduling round the encapsulators end up with partially filled frames. Before sending these frames the ModCod adaptation mechanism can optionally be applied to maximize the capacity utilization. This algorithm scans all active ModCods starting from the one with the highest spectral efficiency. For those ModCods having a framing efficiency lower than a certain design value it is checked whether the packets within that frame can be packed into other frames of ModCods with lower spectral efficiency with sufficient free space. This way the packets are encapsulated more efficiently, reducing the resources necessary to transmit the selected packets and achieving a trade-off between spectral efficiency and framing efficiency.

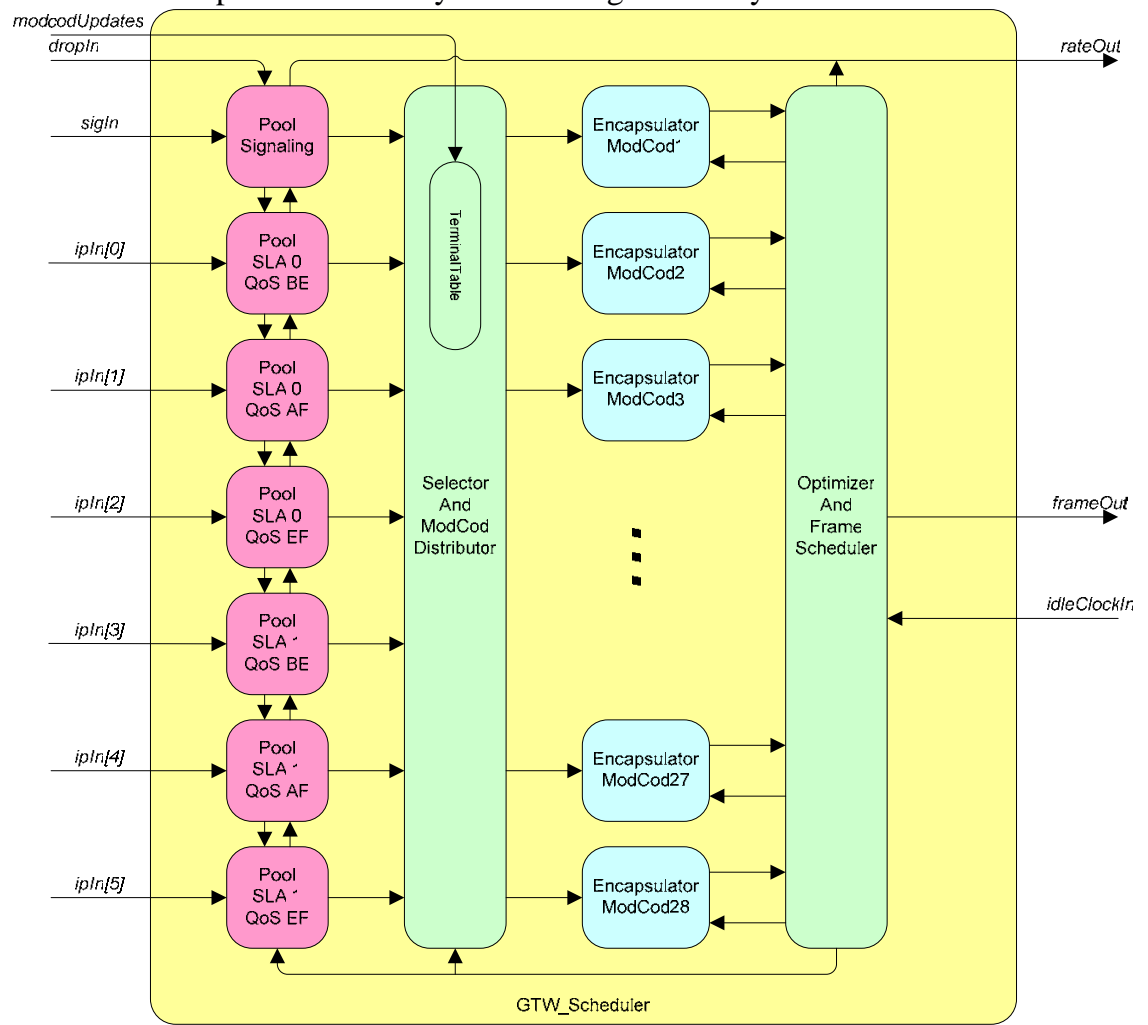

Figure 4: Architecture of packet scheduler in the forward link.

\section{Simulator Architecture}

\section{A. Overall Architecture}

The simulator consists of three large blocks: pre-processing, system simulation and post-processing. Preprocessing computes Signal-to-Noise-and-Interference (SNIR) time series and ModCod selection thresholds. This computation is implemented as a series of Matlab scripts. The result of the pre-processing is a number of large datafiles that are used by the system simulation. The system simulation is implemented in OMNeT++ as a discrete event simulation. ${ }^{6}$ The OMNeT++ simulator produces a vector file which contains measurement vectors. This file is then used by another set of Matlab scripts for post-processing. The configuration of the simulator is done using XMLfiles which are usable from both Matlab and OMNeT++. A screenshot of the graphical user interface of the simulator is shown in Figure 5. 


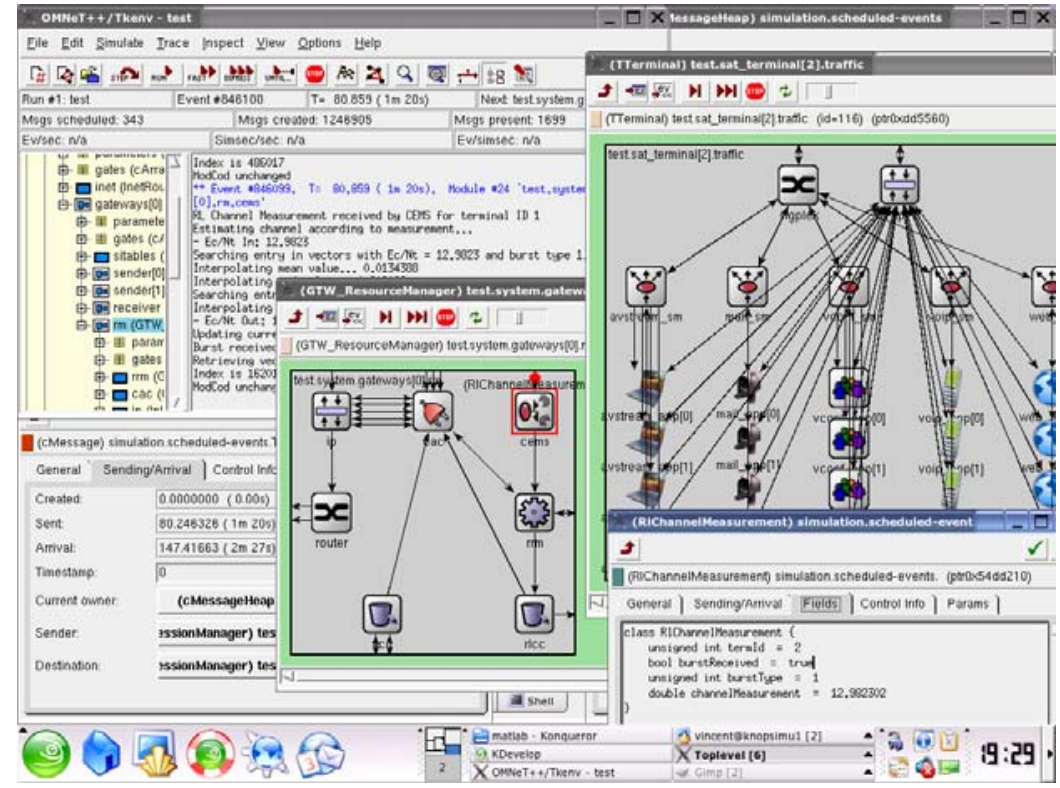

Figure 5. Screenshot of the simulator graphical user interface.

The top-level structure of the system simulator is shown on the left side of Figure 6. The central module called 'System' contains the DVB-S2/RCS gateways and the Internet. Connected to this module are a number of internet terminals, which consist mainly of traffic models and an IP-layer. On the other side the system module is connected to a number of forward link transponders and return link transponders.
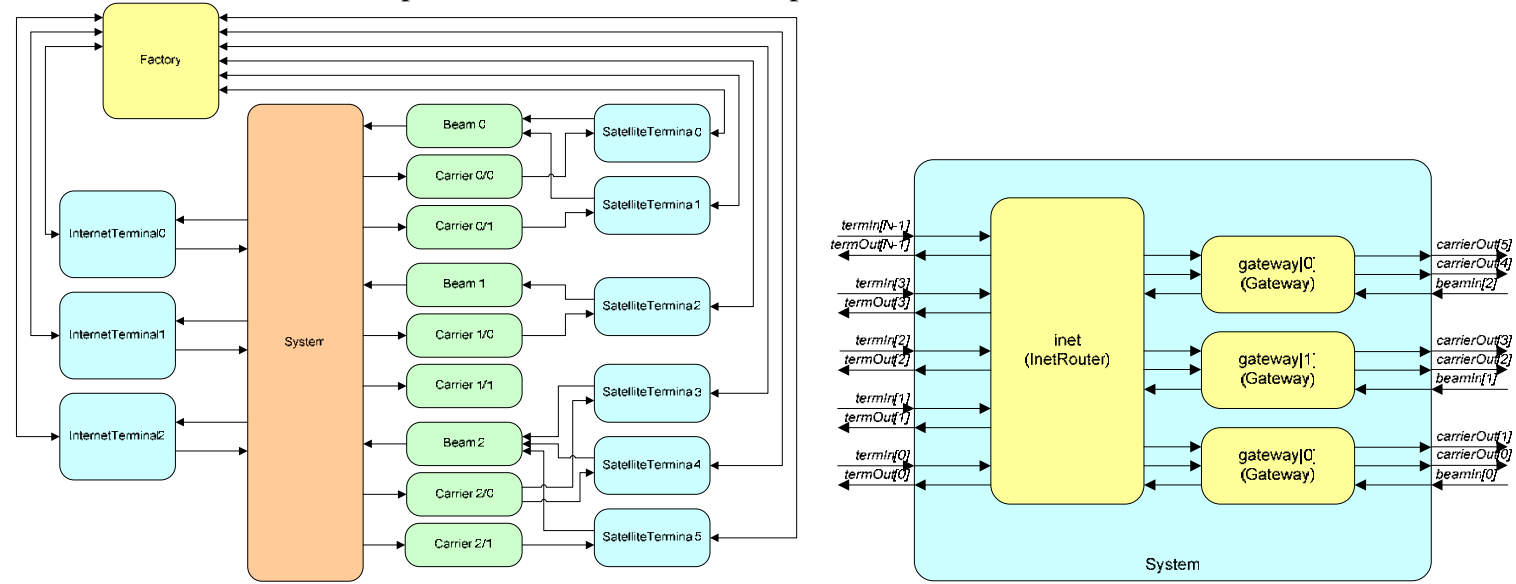

Figure 6: Top-level system simulator block diagram

The number of return link transponders corresponds to the number of gateways in the system - each of these transponders handles multiple return link carriers. Each gateway is also connected to a configurable number of forward link carriers. This allows the simulation of systems which uses multiple forward link carriers. The satellite terminals are connected to one of the forward link carriers of a given gateway (to receive the DVB-S2 forward traffic) and to the return link transponder of the same gateway (to transmit the DVB-RCS return traffic). The mapping of satellite terminals to gateways and forward carriers is fixed for a given simulation and is described in the XML configuration file. In addition to the gateways the system module contains an IP router which represents the terrestrial internet.

The number of terminals, gateways, carriers and other parameters are all specified in the XML configuration file. Therefore most of the modules of the simulator are created dynamically at runtime. The only static module is the so called factory module. This module has two tasks: (1) it reads the XML configuration and creates the system module, the transponders, the internet terminals and the satellite terminals, connecting these together and holding a connection to each terminal; and (2) handling a session protocol for the simulated applications using the connections to the terminals that ensures that the configured traffic parameters are met. While instantiating modules the factory 
retrieves module parameters from the XML file and attaches these parameters to the modules. This technique allows great flexibility and will allow dynamic terminal creation in the future (for simulation of logon/logoff).

\section{B. Gateway}

The gateway is composed of four main blocks: the sending path (transmitter), the receive path (receiver), the resource management (RM) and the SI tables (cf. Figure 7).

The sending path (at the bottom) receives the packets issued from the different applications run by the users which are located in the internet terminals behind the gateway. These IP packets enter into the interworking function and policy enforcement point module (IWF PEP) which is responsible for classifying them according to their QoS classes and for regulating the traffic (controllable rates according to the SLA of the destination terminal for each traffic class). The IP packets are then sent to the scheduler who will schedule them according to different levels of priority and the current estimated ModCod for each terminal and hand them over to the layer 2. The layer 2 will fragment and encapsulate the IP packets according to the method selected (MPE, GSE). The resulting packets are then forwarded to the input stream adapter. This module will produce BBFrames which the S2 Modem turns into physical frames.

On the other hand, the receive path (at the top) is composed of a terminal decoder which will decode the physical frames, introducing the SNIR time series generated previously and forwarding the necessary information to the CEMS module, checking for slot collisions, and forwarding the defragmented packets to the layer 2 module and the signalling to SI Tables module. The layer 2 module decapsulates the packets. The layer 3 interworking function module (L3 IWF) is in charge of redirecting the CAC messages to the RM block and the traffic to the terminals.

The resource management block (in the middle) is composed of several modules: an IP layer and a router to respectively decapsulate/encapsulate the CAC messages and redirect them to the correct carrier (sending path), a Call Admission Control module (CAC, for VoIP), congestion control modules for both the forward- and the returnlink (respectively FLCC and RLCC), a Return-link Resource Manager (RRM) and a Channel Estimator and ModCod Selector (CEMS). The CEMS module is responsible for estimating the current channel condition according to the channel measurements transmitted by the terminal decoder and for selecting the appropriate ModCod.

The SI composition tables module (in the middle) receives the Terminal Burst Time Plan (TBTP) and the Composition Tables (CT), which include the SuperFrame composition and terminal distribution for the Return Link, from the RRM module and produces the different L2 signalling messages.

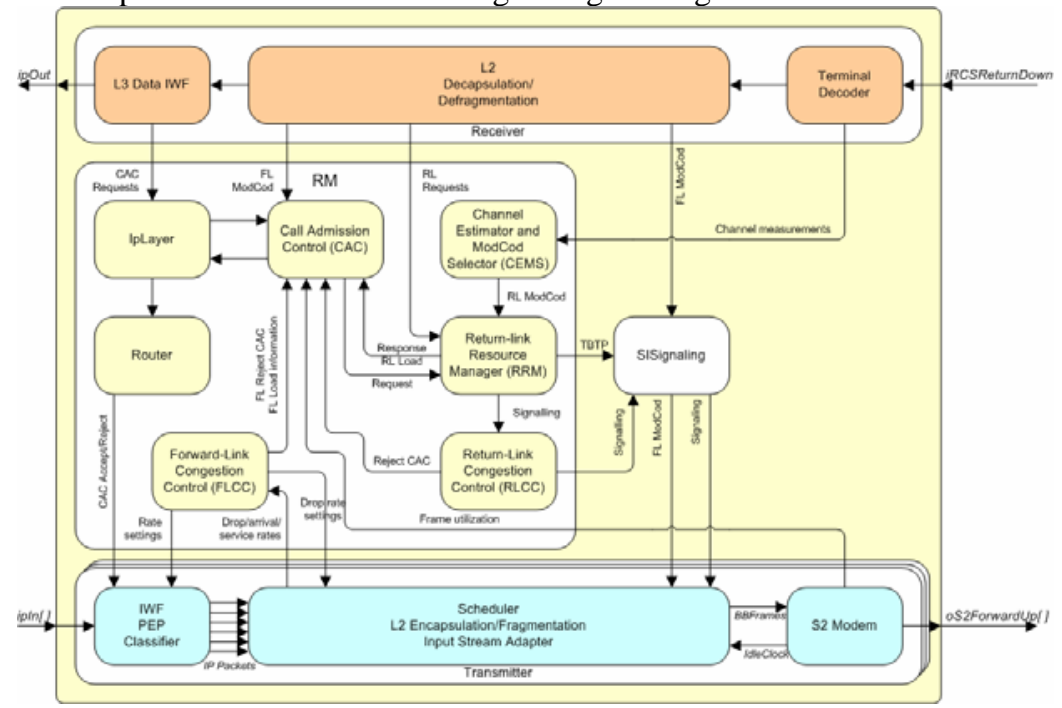

Figure 7: Gateway block diagram

\section{Terminal}

The terminal is composed, as the gateway, of the following four main blocks: the sending path (transmitter), the receive path (receiver), the resource management (RM) and the SI tables (Figure 8).

The sending path (at the bottom) receives the packets issued from the different applications of the users which are connected to the terminal. These IP packets enter into the IWF PEP module which is responsible for classifying them according to their QoS classes and for policing the traffic (controllable rates for each traffic class). The IP packets are then sent to the scheduler who will forward them according to their QoS classes to the MAC layer. The 
MAC layer will fragment and encapsulate the IP packets according to the method selected which comprise MPEG or ATM TRF bursts for the FIL scheme, and a GSE-like protocol for the FBL scheme. The resulting packets are then forwarded to the RCS Modem and turned into physical layer frames. User packets are inserted into the SF according to the allocation received from the gateway RRM via the TBTP.

The receive path (at the top) is composed of a Physical Layer (PL) decoder which will include the previously computed SNIR time Series and forward the resulting BBFrames to the Descapsulator/BBFrame Decoder. At this point the BBFrames will be decoded and the packets (MPE, GSE) will be extracted. The decapsulator reproduces the IP packets and the layer 3 interworking function module (L3 IWF) is in charge of forwarding the IP packets without performing any operation to the applications.

The resource management block (in the middle) is composed of several modules: a congestion control module for the return-link (RLCC), a Request Manager (RM) and a Channel Estimator and Modcod Selector (CEMS). The $\mathrm{RM}$ module analyses the states of the queues located in the scheduler and requests resources to the gateway RRM module.

The SI resource tables module (in the middle) receives the capacity requests and the applicable FL ModCod from the RM module which are then signalled to the gateway RRM via contention based slots or signalling in already allocated TRF bursts. ${ }^{2}$ Further, this module reads the information from the TBTP received from the gateway which is then forwarded to the Encapsulation/Fragmentation MAC where this information is used to correctly distribute the user data and signalling in the SF.

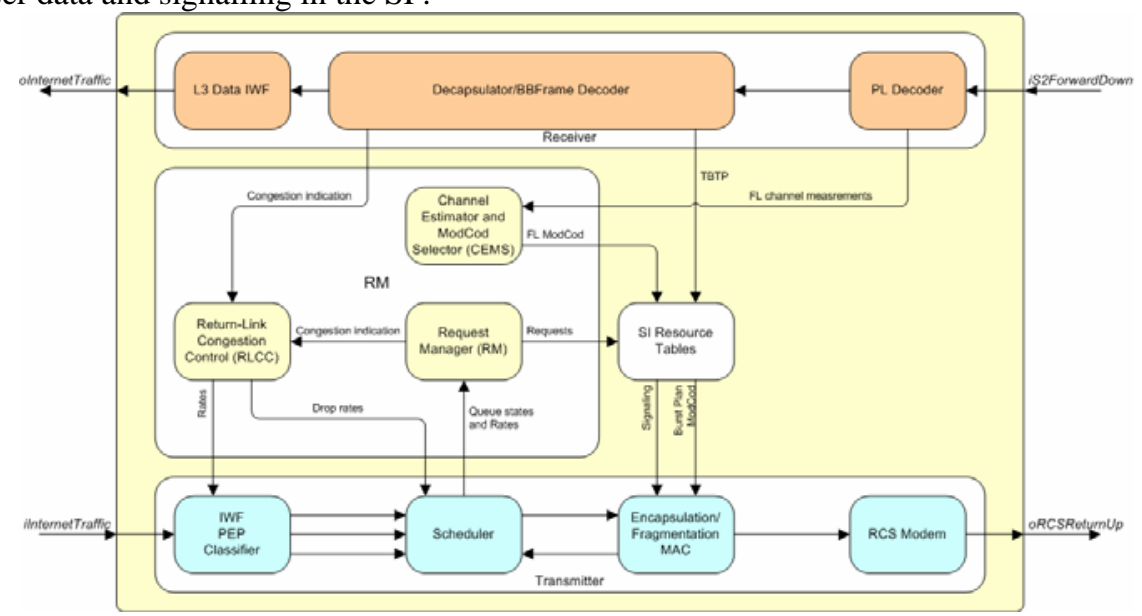

Figure 8: Terminal block diagram

\section{Satellite}

The transparent case is simple because the satellite just relays the signal to the ground after frequency translation and amplification.

Some optimisation in the implementation of the satellite in the simulator has been performed to reduce the number of messages to be handled by the simulator.

\section{Simulations}

\section{A. Simulation Scenario Details}

The particular parameters assumed for the gateway, user terminals, and transparent satellite are shown in Table 1.

\begin{tabular}{|c|c|c|c|c|}
\hline System Parameter & \multicolumn{2}{c|}{ Forward Link } & \multicolumn{2}{c|}{ Return Link } \\
& Feeder Link & User Link & Feeder Link & User Link \\
\hline \hline Carrier frequency & $28.50 \mathrm{GHz}$ & $19.95 \mathrm{GHz}$ & $18.70 \mathrm{GHz}$ & $29.75 \mathrm{GHz}$ \\
\hline Access , Multiplex & TDM, FDM & TDM & MF-TDMA,FDM & MF-TDMA \\
\hline Total bandwidth $B_{\text {tot }}$ & $500 \mathrm{MHz}$ & $500 \mathrm{MHz}$ & $500 \mathrm{MHz}$ & $500 \mathrm{MHz}$ \\
\hline Number of antenna beams & 72 & 72 & 72 & 72 \\
\hline Frequency reuse $f_{R}$ & 1 & 4 & 1 & 4 \\
\hline Bandwidth per beam & $2000 \mathrm{MHz}$ & $125 \mathrm{MHz}$ & $2000 \mathrm{MHz}$ & $125 \mathrm{MHz}$
\end{tabular}




\begin{tabular}{|c|c|c|c|c|}
\hline No. of carriers per beam & $(14-15) * 2$ & 2 & $(14-15) *(20-84)$ & $20-84$ \\
\hline $\begin{array}{c}\text { Carrier Symbol Rate } \\
\text { (SRC filter Roll-off, carrier } \\
\text { spacing) }\end{array}$ & $\begin{array}{l}2 * 45 \text { Msps } \\
(0.25,0.11)\end{array}$ & $\begin{array}{l}2 * 45 \text { Msps } \\
(0.25,0.11)\end{array}$ & $\begin{array}{l}\text { 1,2,4 Msps } \\
(0.35,0.11)\end{array}$ & $\begin{array}{l}1,2,4 \text { Msps } \\
(0.35,0.11)\end{array}$ \\
\hline Number of Gateways & 5 & - & 5 & - \\
\hline Polarisation reuse $N_{p}$ & 1 & 1 & 1 & 1 \\
\hline Satellite $\mathrm{Rx} / \mathrm{Tx}$ antenna peak gain & $\approx 54 \mathrm{dBi}$ & $\approx 54 \mathrm{dBi}$ & $\approx 54 \mathrm{dBi}$ & $\approx 54 \mathrm{dBi}$ \\
\hline Satellite antenna loss (efficiency) & $1 \mathrm{~dB}$ & $1 \mathrm{~dB}$ & $1 \mathrm{~dB}$ & $1 \mathrm{~dB}$ \\
\hline $\begin{array}{c}\text { Sat. RF Tx saturated power per } \\
\text { UL/GW beam }\end{array}$ & - & $\begin{array}{c}15 \text { dBW per } 45 \\
\text { Msps } \\
\end{array}$ & $\begin{array}{c}\text {-10.4 dBW per } 1 \\
\text { Msps } \\
\end{array}$ & - \\
\hline Satellite HPA Output back-off & - & $\begin{array}{l}\text { (taken into } \\
\text { account in SNR } \\
\text { Req.) } \\
\end{array}$ & $4 \mathrm{~dB}$ & - \\
\hline Satellite Output loss & & $2.85 \mathrm{~dB}$ & $1.75 \mathrm{~dB}$ & \\
\hline Satellite G/T (COC) & $24.6 \mathrm{~dB} / \mathrm{K}$ & - & - & $24.6 \mathrm{~dB} / \mathrm{K}$ \\
\hline Satellite noise temperature & $28.4 \mathrm{dBK}$ & & & $28.4 \mathrm{dBK}$ \\
\hline GW Tx antenna peak gain & $65.2 \mathrm{dBi}$ & - & - & - \\
\hline GW RF Tx total max./nom. power & $\begin{array}{c}18 \text { dBW for } 8 \text { carr } \\
\text { (=1 cluster })\end{array}$ & - & - & - \\
\hline GW Output back-off & $6.0 \mathrm{~dB}$ & & & \\
\hline GW Output loss & $2.0 \mathrm{~dB}$ & & & \\
\hline GW Pointing loss & $1.1 \mathrm{~dB}$ & & & \\
\hline GW G/T (clear-sky) & - & - & $37.9 \mathrm{~dB} / \mathrm{K}$ & - \\
\hline GW clear sky temperature & - & - & $23.6 \mathrm{dBK}$ & - \\
\hline $\begin{array}{c}\text { UT Tx antenna peak gain } \\
\text { (0.75 m diameter, 60\% efficiency) }\end{array}$ & - & - & - & $\begin{array}{l}\text { 45.2, } 48.1,49.2 \\
\mathrm{dBi} \\
\end{array}$ \\
\hline UT RF TX max./nom. power & - & - & - & $4.0,4.0,6.0 \mathrm{dBW}$ \\
\hline UT Output back-off & & & & $0.5 \mathrm{~dB}$ \\
\hline UT Output loss & & & & $0.5 \mathrm{~dB}$ \\
\hline UT Pointing loss & & & & $1.0 \mathrm{~dB}$ \\
\hline UT G/T (clear-sky) & - & $\begin{array}{c}\text { 17.2, 20.1, } 21.3 \\
\mathrm{~dB} / \mathrm{K}\end{array}$ & - & - \\
\hline UT clear-sky temperature & - & $24.5 \mathrm{dBK}$ & - & - \\
\hline Rain temperature delta & - & $23.5 \mathrm{dBK}$ & $23.5 \mathrm{dBK}$ & - \\
\hline
\end{tabular}

Table 1: Transparent scenario gateway, user terminal, and satellite parameters

An important factor that needs to be considered in the simulations is interference:

- Co-channel interference (CCI) being caused by the re-use of the same frequency band and polarisation in several beams of the satellite system,

- $\quad$ adjacent channel interference (ACI),

- $\quad$ inter-modulation (IM) interference, and

- interference from other systems.

Table 2 presents the carrier-to-interferences ratios (C/I) for the various interference contributions.

\begin{tabular}{|c|c|c|c|c|}
\hline \multirow{2}{*}{ Interference } & \multicolumn{2}{|c|}{ Forward Link } & \multicolumn{2}{|c|}{ Return Link } \\
\hline & Feeder Link & User Link & Feeder Link & User Link \\
\hline CCI (co-polar) & \multicolumn{4}{|c|}{ (is calculated as part of pre-calculations) } \\
\hline$(\mathrm{C} / \mathrm{I})_{\mathrm{ACI}}$ & - & - & $\begin{array}{l}10 \% \text { noise } \\
\text { increase wrt. } \\
\text { white noise }\end{array}$ & $17 \mathrm{~dB}$ \\
\hline$(\mathrm{C} / \mathrm{I})_{\mathrm{IM}}$ & $\begin{array}{c}\approx 27 \mathrm{~dB}(\mathrm{NPR}= \\
25 \mathrm{~dB}, \text { non-lin } \\
\text { HPA })\end{array}$ & - & $\begin{array}{c}\approx 22 \mathrm{~dB}(\mathrm{NPR}= \\
18 \mathrm{~dB}, \operatorname{lin} . \mathrm{HPA})\end{array}$ & - \\
\hline
\end{tabular}




\begin{tabular}{|c|c|c|c|c|}
\hline $\begin{array}{c}\text { external noise power density, } \\
\text { other satellite system } \\
\text { interference }\end{array}$ & $-207 \mathrm{dBW} / \mathrm{Hz}$ & $\begin{array}{c}\mathrm{C} / \mathrm{I}=24.7,27.7, \\
28.8 \mathrm{~dB}\end{array}$ & - & $\begin{array}{c}\mathrm{C} / \mathrm{I}=27.5,30.4, \\
31.6 \mathrm{~dB}\end{array}$ \\
\hline
\end{tabular}

Table 2: Transparent scenario interference parameters

\section{B. Results}

First results have been obtained during testing of the simulator. The simulations use a small transparent scenario composed of 4 satellite 1MSymbol/s-terminals, 8 internet terminals, one beam with two forward link carriers. The traffic generated with this setup is well below the system capacity so that there is no congestion and no rejects from call admission control. The traffic resulting from this setup is shown in Figure 9. There are five types of applications: web traffic, POP3 mail traffic, VoIP (G.723 codec), Audio/Video streaming and video conferencing. The parameters for these models are configurable via the XML configuration file. Because of the short simulation time show in Figure 9 not all applications can be seen. The peaks for QoS0 are GET responses from web sessions which generated traffic in QoS class 0 (best effort). The light blue line is a VoIP session in SLA class gold and QoS class 2 (expedited forwarding). There is no traffic in QoS class 1 (assured forwarding) during this simulation.

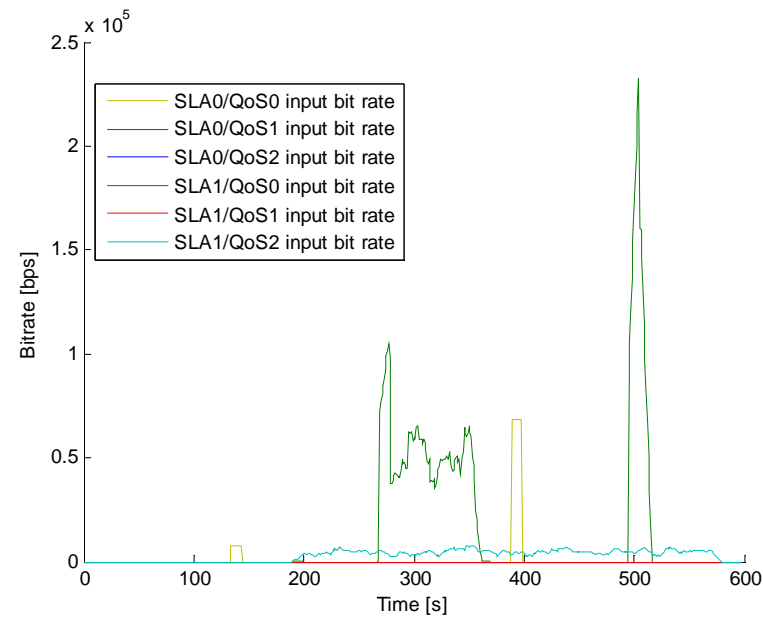

Figure 9: Input bit rates of the forward link scheduler

The resulting utilization of the S2-Modem (forward link) is shown in Figure 10. The frame utilization is the percentage of time during which non-dummy frames are sent. It does not account for the filling state of the frames. The straight line up to 180 seconds shows the percentage of physical layer frames that carry signalling traffic. It must be noted, that the signalling capacity used is not $3 \%$ because frames carrying signalling are not necessarily completely filled. Starting from 180 seconds the frames filled with useful traffic can be seen which correlates with the forward link scheduler input in Figure 9.

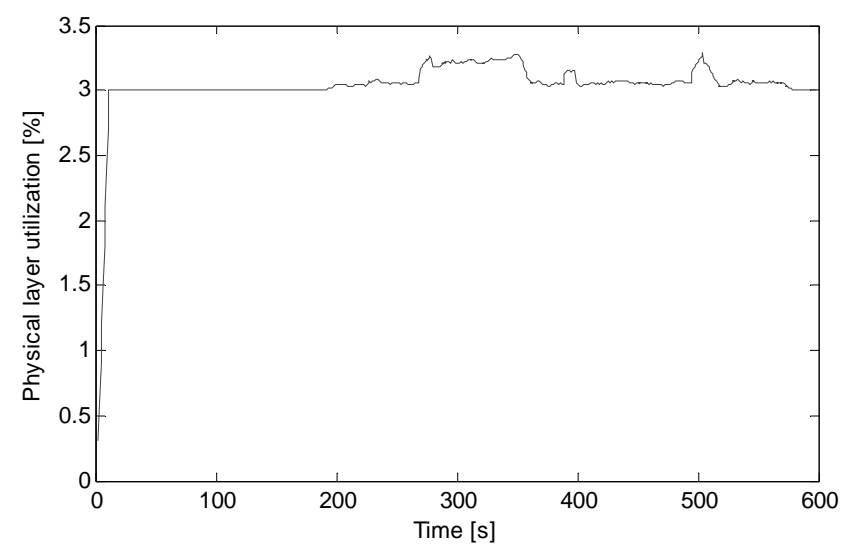

Figure 10: Physical layer frame utilization in the forward link modem 
Figure 11 and Figure 12 show the delay jitter CDF and PDF for the VoIP application for the forward respectively the return link. The delay jitter is measured as the inter-arrival time between consecutive packets compared to the expected inter-arrival time. For the given configuration of the VoIP model with G.723 codec one 100-byte IP packet containing two codec frames of 30 bytes is sent every $60 \mathrm{~ms}$. In the forward link one single peak can be seen which (in the almost idle system) is caused by the scheduling algorithm and the physical layer framing. The mean value of the jitter is $2.4 \mathrm{~ms}$ with a standard deviation of $0.75 \mathrm{~ms}$.

On the return link there are two peaks in the jitter PDF. These are caused by the different periodicity of the application and the DVB-RCS superframe. The application sends a packet every $60 \mathrm{~ms}$ whereas the superframe is $50 \mathrm{~ms}$ long. Because there is a maximum of one VoIP packet per superframe, the jitter optimization of the return link resource management algorithm does not help in this case. On the return link the mean jitter is $23 \mathrm{~ms}$ with a standard deviation of $3.8 \mathrm{~ms}$.
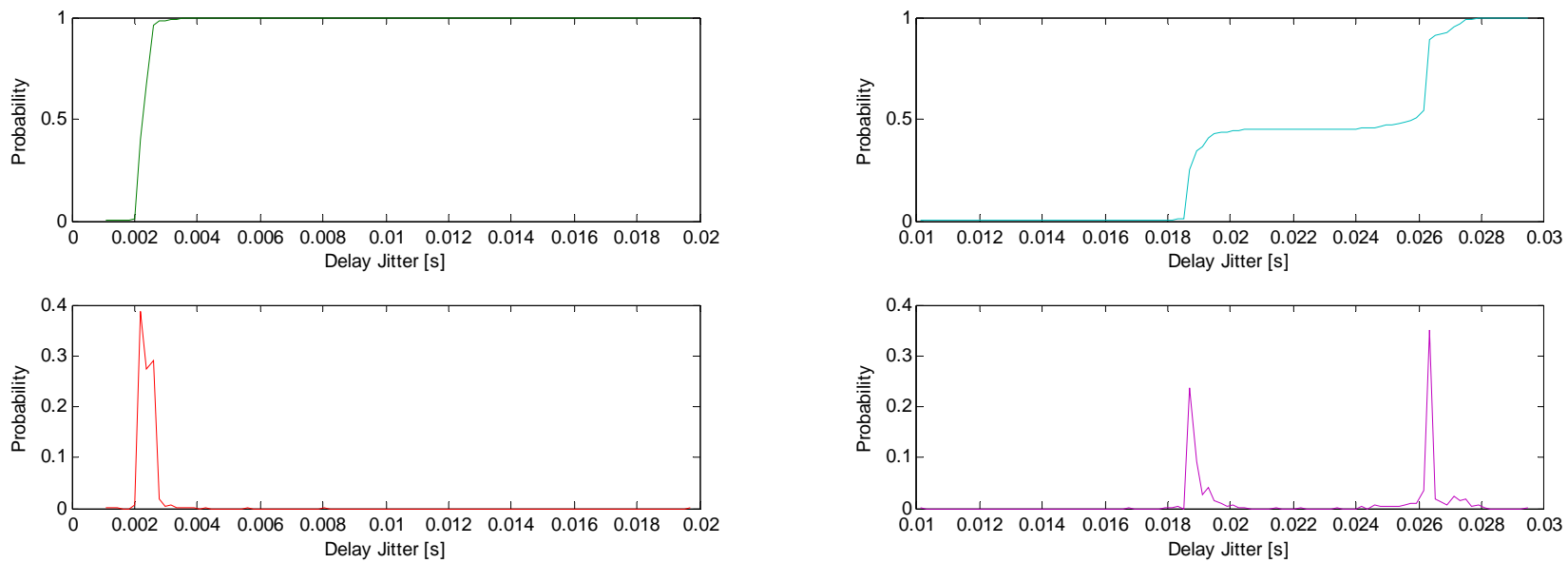

Figure 11. VoIP delay jitter in the forward link (top: CDF; bottom: PDF).

Figure 12. VoIP delay jitter in the return link (top: CDF; bottom: PDF).

Figure 13 shows the working of the ModCod selection algorithm for the forward link. In the upper graph the output of the channel estimator in the terminal is shown. The channel estimator periodically computes a C/ $\mathrm{N}_{0}$ value that is used by ModCod selector to select an appropriate ModCod to be used in the forward link. The selected ModCod is then signalled to the gateway. The green lines are the thresholds for switching from a lower to a higher ModCod while the red lines are the thresholds for switching from a higher ModCod to a lower one. These are different to implement a hysteresis that prevents ModCod thrashing due to scintillation effects.

The result of the ModCod selection is shown in the lower part. It can be seen that at time 20700 a fade event of $3 \mathrm{~dB}$ starts that causes the ModCod selector to switch from ModCod 14 down to 10 in several steps. When the fade goes away the selector switches back to the higher ModCods. 

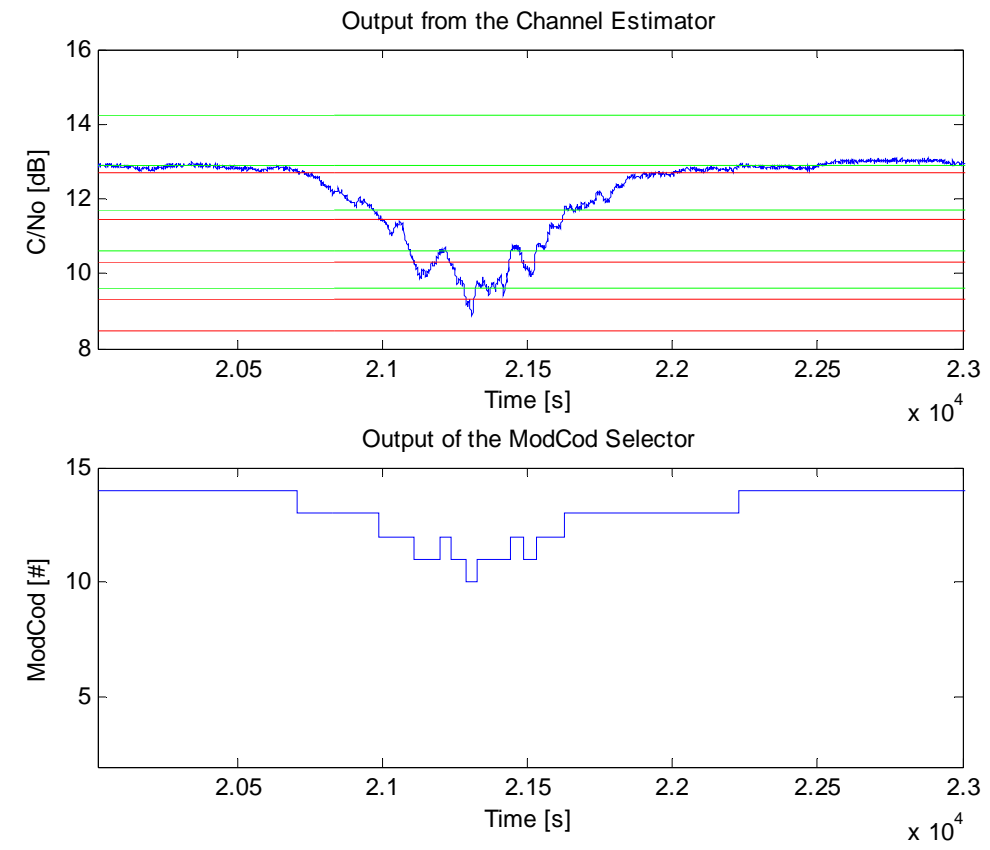

Figure 13. Outputs of the forward link channel estimator and ModCod selector module.

The $\mathrm{C} / \mathrm{N}_{0}$ time series are computed during the pre-processing phase. A channel simulation tool implemented in Matlab computes spatial correlated rain fading, scintillation effects, estimates external and intra-system interferences and noise and calculates signal to noise and interference ratio time series for the forward and return down and uplinks depending on terminal position.

\section{Acknowledgments}

The authors are grateful to many colleagues for intensive discussions and support in various activities in which this work was developed. The work on GSE encapsulation started in an ESA contract and is now in the standardisation process with ETSI and the DVB-GBS work group. The contributions and discussions with Oscar Del Rio (ESA) are gratefully acknowledged.

\section{References}

${ }^{1}$ Digital Video Broadcasting (DVB): "Second generation framing structure, channel coding and modulation systems for Broadcasting, Interactive Services, News Gathering and other broadband satellite applications”, European Standard (Telecommunications series), ETSI EN 302 307, V1.1.2, 2006.

${ }^{2}$ Digital Video Broadcasting (DVB): “Interaction Channel for satellite distribution system”, European Standard (Telecommunications series), ETSI EN 301790 v1.4.1, 2005-09.

${ }^{3}$ R. Rinaldo, R. De Gaudenzi, "Capacity analysis and system optimization for the forward link of multi-beam satellite broadband systems exploiting adaptive coding and modulation," International Journal of Satellite Communications and Networking, pp. 401-423, vol. 22, issue 3, Jun. 2004

${ }^{4}$ Audens ACT GmbH, TriaGnoSys GmbH, "Protocols and Signalling for Adaptive Fade Mitigation Techniques (FMT) in DVB-RCS Multi-Beam Systems”, Final Report, ESA Study 17403/03/NL/ND, 2004.

${ }^{5}$ O. Lücke, A. Jahn, M. Werner, “MAC and encapsulation efficiency of satellite DVB using fade mitigation techniques”, in International Journal of Satellite Communications and Networking, vol. 24, iss. 6, Nov./Dec. 2006, pp. 521-559

${ }^{6}$ A. Varga, "The OMNET++ Discrete Event Simulation System", Proceedings of the European Simulation Multiconference (ESM’2001), June 6-9, 2001. Prague, Czech Republic.

${ }^{7}$ C. Párraga Niebla, “Scheduling Techniques for Satellite Systems with Adaptive Coding and Modulation”, in Proc. of ASMS Conference, 29 - 31 May 2006, Herrsching, Germany. 